\title{
Endoluminal Reconstruction for Nonsaccular Aneurysms of the Proximal Posterior Cerebral Artery with the Pipeline Embolization Device
}

\author{
D.W. Zumofen, M. Shapiro, T. Becske, E. Raz, M.B. Potts, H.A. Riina, and P.K. Nelson
}

\begin{abstract}
BACKGROUND AND PURPOSE: Treatment options for nonsaccular posterior cerebral artery aneurysms include a range of surgical and endovascular reconstructive and deconstructive methods. However, no truly satisfactory treatment option is available to date for lesions arising from the $\mathrm{Pl}$ and $\mathrm{P} 2$ segments. The purpose of the present case series is to investigate both the efficacy and safety of the Pipeline Embolization Device in treating these challenging aneurysms.
\end{abstract}

MATERIALS AND METHODS: We present a series of 6 consecutive patients who underwent endoluminal reconstruction with the Pipeline Embolization Device for nonsaccular P1 or P2 segment aneurysms between January 2009 and June 2013.

RESULTS: Aneurysm location included the P1 segment in 2 patients and the $\mathrm{P} 2$ segment in 4 patients. Mean aneurysm diameter was $23 \mathrm{~mm}$ (range, 5-44 mm). Mean length of the arterial segment involved was $10 \mathrm{~mm}$ (range, 6-19 mm). Clinical presentation included mass effect in 4 patients and perforator stroke and subacute aneurysmal subarachnoid hemorrhage in 1 patient each. Endovascular reconstruction was performed by using 1 Pipeline Embolization Device in 5 patients and 2 overlapping Pipeline Embolization Devices in the remaining patient. Angiographic aneurysm occlusion was immediate in 1 patient, within 6 months in 4 patients, and within 1 year in the remaining patient. Index symptoms resolved in 4 patients and stabilized in the remaining 2. No new permanent neurologic sequelae and no aneurysm recurrence were recorded during the mean follow-up period of 613 days (range, 540-725 days).

CONCLUSIONS: Endovascular reconstruction with the Pipeline Embolization Device for nonsaccular aneurysms arising from the P1 and P2 segments compares favorably with historical treatment options in terms of occlusion rate, margin of safety, and neurologic outcome.

ABBREVIATIONS: aSAH = aneurysmal subarachnoid hemorrhage; $\mathrm{CN}=$ cranial nerve; $\mathrm{PCA}=$ posterior cerebral artery; $\mathrm{PED}=$ Pipeline Embolization Device

$\mathbf{N}$ onsaccular posterior cerebral artery (PCA) aneurysms comprise a spectrum of arterial wall diseases spanning a variety of often complex, partially thrombosed lesions with holosegmental involvement as a common attribute. ${ }^{1}$ Proposed etiologies include congenitally derived lesions, atherosclerosis, and arterial wall dissections of idiopathic, inflammatory, or traumatic origin. ${ }^{2}$ Whereas the dissecting subset may have a tendency to present

Received August 6, 2014; accepted after revision November 17.

From the Bernard and Irene Schwartz Interventional Neuroradiology Section, Department of Radiology (D.W.Z., M.S., T.B., E.R., M.B.P., H.A.R., P.K.N.), and Departments of Neurological Surgery (D.W.Z., M.B.P., H.A.R., P.K.N.) and Neurology (M.S. T.B.), New York University School of Medicine, New York, New York.

Daniel W. Zumofen has a personal scholarship from the Fund Helmut Hartweg for the promotion of young researchers in the field of medical radiology and the Schweizerische Akademie der Medizinischen Wissenschaften.

Please address correspondence to Peter Kim Nelson, MD, Bernard and Irene Schwartz Interventional Neuroradiology Section, Department of Radiology, New York University School of Medicine, 560 First Ave, 7th floor, New York, NY 10016; e-mail: peter.nelson@nyumc.org

$\equiv$ Indicates article with supplemental on-line tables.

http://dx.doi.org/10.3174/ajnr.A4261 early with acute hemorrhagic or ischemic stroke, the remaining aneurysms typically come to clinical attention later in the course of their development and hence at comparatively large dimensions. ${ }^{3}$ Nondissecting, nonsaccular PCA aneurysms have a predilection for $\mathrm{P} 1$ and $\mathrm{P} 2^{4}$ segments and have a tendency to have a sizable thrombotic subcompartment.,

The infrequency of these lesions has relegated the surgical and endovascular experience to a relatively thin body of literature. ${ }^{1,5,7-15}$ Reported treatment strategies include a range of reconstructive and deconstructive methods, often distinguished by their variability in outcome (On-line Table 1). We now report and discuss our experience with use of the Pipeline Embolization Device (PED; Covidien, Irvine, California) to treat a series of 6 consecutive cases of complex aneurysms arising from the P1 or P2 segment.

\section{MATERIALS AND METHODS}

Six consecutive patients with nonsaccular aneurysms (circumferentially involving the parent vessel) of the P1 and P2 segments 
underwent endovascular reconstruction with the PED by our team between January 2009 and June 2013. Basilar apex aneurysms involving the $\mathrm{P} 1$ segment were excluded from the present series because in our opinion, they represent a different disease with a very different set of challenges. Patient characteristics are provided in On-line Table 2. The average aneurysm diameter was $23 \mathrm{~mm}$ (range, 5-44 mm). The mean length of the arterial segment involved was $10 \mathrm{~mm}$ (range, 6-19 mm). Five aneurysms were unruptured, and 1 came to our attention several months following low-grade aneurysmal subarachnoid hemorrhage (aSAH) related to the index $\mathrm{P} 1$ aneurysm - initially managed with observation at an outside institution. Index symptoms of the 5 patients with unruptured aneurysms included mass effect-related compression symptoms in 4 patients and hemiparesis following a thalamic perforator stroke in the remaining patient. Mass effectrelated compression symptoms included cranial nerve $(\mathrm{CN})$ III palsy in 2 patients, sensorimotor hemisyndrome combined with $\mathrm{CN}$ III palsy in 1 patient, and dizziness in 1 patient. Four of the 6 aneurysms included in the present series showed sizable (subtotal) thrombotic subcompartment, 1 showed minimal thrombosis, and the remaining one showed no evidence of thrombosis.

All patients were pretreated with acetylsalicylic acid, $325 \mathrm{mg}$ daily, and clopidogrel, $75 \mathrm{mg}$ daily, for at least 5 days. A P2Y12 assay (VerifyNow; Accumetrics, San Diego, California) was obtained at the beginning of the procedure and thereafter daily until discharge to evaluate and confirm the level of platelet inhibition obtained by the dual antiplatelet regimen. Endoluminal reconstruction was performed in all except 1 case by using a single PED. In patient 4 , we elected to overlap 2 devices to maintain single coverage of the normal vascular segments proximal and distal to the aneurysm, with double-coverage of the aneurysm neck. The specific dimensions of the PED were chosen after determination of the length of the aneurysmal arterial segment and parent vessel diameter at the landing zones, proximal and distal to the aneurysm. ${ }^{16}$ The Marksman (Covidien) microcatheter was used for all embolizations, with a variety of proximal support systems. In cases in which distal access with the Marksman could not be primarily established, microcatheterization was performed with an Excelsior SL-10 microcatheter (Stryker, Kalamzoo, Michigan), followed by an over-the-wire exchange for a Marksman by using a Transend Floppy 0.014 300-cm wire (Stryker). PEDs were deployed by using previously described methods of unsheathing and delivery-wire advancement. ${ }^{17}$ An immediate postprocedure noncontrast-enhanced head CT scan was routinely performed. In the absence of intracranial hemorrhage, IV heparin infusion was continued for at least 12 hours at rate of 500-700 IU/h.

Clinical follow-up was performed at 1 month, 6 months, and annually thereafter. Follow-up DSA was performed at 6 months and 1 year. Continued growth of the aneurysmal mass despite angiographic "cure" was ruled out in all cases clinically by regression of mass effect-related symptoms when present and was confirmed by serial transaxial follow-up imaging (CT or MR imaging). With the exception of 1 patient whose clopidogrel therapy was prematurely interrupted at 4 months post-PED to allow performance of urgent abdominal surgery unrelated to the aneurysm, dual antiplatelet inhibition was continued for 12 months, with acetylsalicylic acid therapy maintained indefinitely.

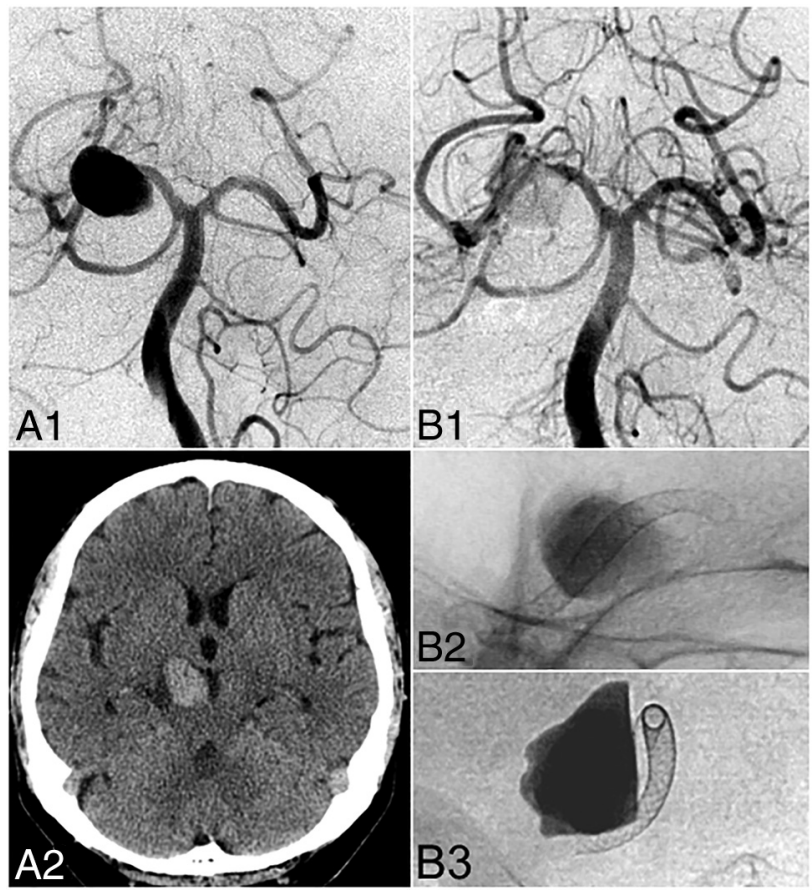

FIG 1. Illustrative case (patient 4). A 68-year-old man presented with right oculomotor nerve palsy. DSA (Al) and noncontrast head CT (A2) reveal a fusiform $\mathrm{P} 2$ aneurysm with a maximum diameter of $23 \mathrm{~mm}$ and a 7-mm length of the arterial segment involved (neck width). Endovascular reconstruction with 2 overlapping PED devices $(2.5 \times 20$ $\mathrm{mm}, 2.75 \times 10 \mathrm{~mm}$ ) led to considerable stasis of contrast in the aneurysm body illustrated on postembolization control DSA (BI) and unsubtracted angiography (B2 and B3).

\section{RESULTS}

The results are listed in On-line Table 2. Angiographic aneurysm occlusion was immediate in 1 patient, within 6 months in 4 patients, and within 1 year in the remaining patient. In the patient requiring clopidogrel discontinuation to allow abdominal surgery, the parent artery was occluded at 6-month angiographic follow-up, without clinical or radiographic evidence of infarction. Index symptoms resolved in 4 of 6 patients and stabilized in the remaining 2. Patients presenting with subacute aSAH (patient 1) and a small mesencephalic perforator stroke (patient 2) recovered from their index symptoms. Mass effect-related index symptoms resolved in 2 of 4 patients and stabilized in the remaining 2. Third cranial nerve palsy (patients 4 and 6) completely resolved. In the 2 patients who presented with symptoms related to long-standing brain stem compression (patients 3 and 5), index symptoms stabilized, but did not resolve. There were no instances of new permanent neurologic deficits during the entire mean of 613 days (range, 540-725 days) of clinical follow-up. An illustrative case (patient 4) is shown in Figs 1 and 2.

Delivery of the PED in patient 3 required advancement of a $5 \mathrm{~F}$ Navien (Covidien) intracranial support catheter into the distal basilar artery and was associated with perforation of the left P1, documented by slow contrast extravasation on DSA. PED deployment was completed across the aneurysm neck and perforation site, leading to immediate hemostasis. Postprocedure non-contrast-enhanced head CT demonstrated subarachnoid blood and contrast within the basal cisterns and the medial left Sylvian fissure. The patient, who initially presented with mass 


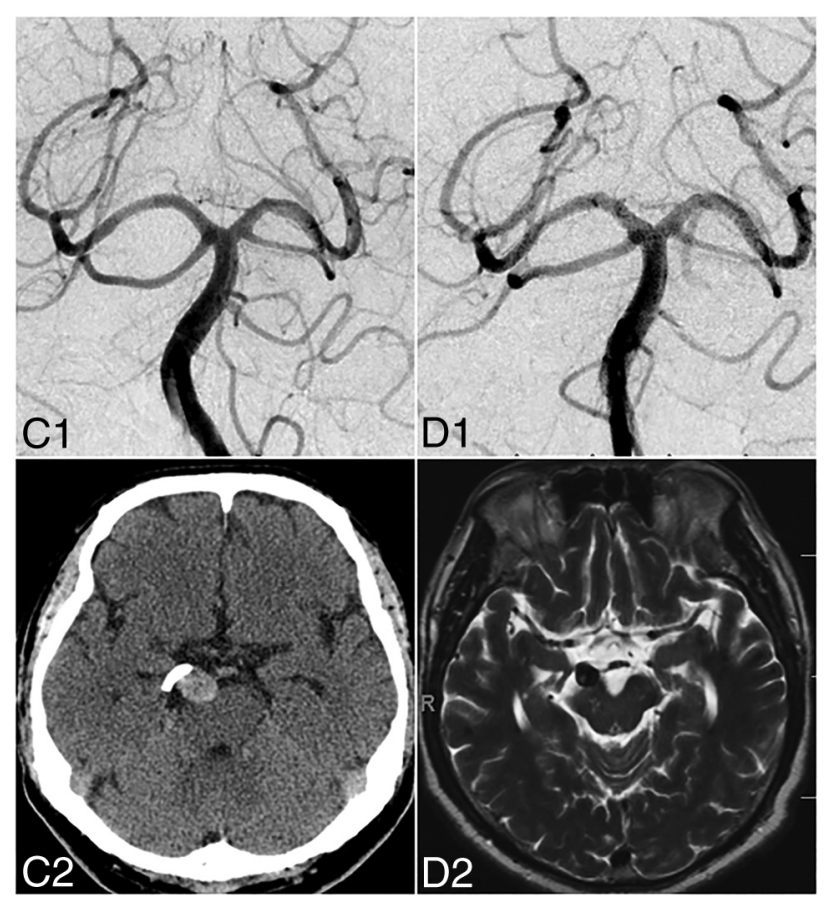

FIG 2. In the same patient, at 6 months, DSA shows complete angiographic aneurysm occlusion (C7). Regression of mass effect-related symptoms (CN III palsy) is correlated with the regression of aneurysm mass effect as illustrated on 6-month follow-up noncontrast head CT (C2). At 1 year, DSA (DI) confirms stable angiographic cure, and axial T2-weighted MR imaging (D2) shows nearly complete involution of the aneurysm sac.

effect symptoms of dizziness and right-sided sensorimotor deficits, emerged from anesthesia with a new headache and worsened right-sided sensorimotor symptoms. She recovered to her preprocedural baseline neurologic status during the next several weeks, without additional endovascular or surgical intervention.

\section{DISCUSSION}

The advent of minimally porous endoluminal devices has produced a paradigm shift in the treatment of both anterior and posterior circulation aneurysms ${ }^{17,18}$ and, in particular, has generated enthusiasm for their use in challenging posterior circulation lesions. Although the early experience with flow diverters in the posterior circulation has been mixed, ${ }^{9,19}$ the variability in outcomes likely reflects, in part, the heterogeneity of lesions involving the posterior circulation group rather than an inherent limitation of the treatment technique per se. The current small series illustrates this point, demonstrating both the efficacy and reasonable safety of the PED in treating proximal PCA aneurysms. This is further underscored by the large size and complex geometry of lesions in this series and by the single technical complication related to device deployment in a case of particularly challenging geometry.

For the most part, treatment with the PED was limited to a single device due to concern for coverage of eloquent regional perforators. This was circumvented in patient 4 , in whom it was elected to overlap 2 shorter devices across the aneurysm neck in a manner that maintained single coverage of the normal vascular segments proximal and distal to the aneurysm, illustrating one technical refinement to enable increased selective coverage of the aneurysm, while concomitantly minimizing the associated coverage risk to adjacent perforators. ${ }^{20}$ Given reports of occasional late in-stent thrombosis, particularly in the posterior circulation, ${ }^{21}$ it may be prudent to prolong dual antiplatelet therapy (12 months or longer) and to consider indefinite single agent maintenance. We think that the single incidence of in-stent thrombosis in our series upon after required deliberate discontinuation of clopidogrel clearly supports such and antiplatelet regimen.

In line with previously reported results for endoluminal reconstruction by using the PED for large and giant aneurysms in the anterior circulation, ${ }^{22} 2$ of 4 patients who presented with long-standing neural compression symptoms did not experience full clinical recovery despite documented aneurysm involution. In our opinion, these results are largely in line with those that would be expected after classic deconstructive treatment such as proximal parent artery clip or coil occlusion. In the present series, transaxial follow-up imaging was performed in all cases, demonstrating, in line with previous reports, ${ }^{22,23}$ an acute stage of thrombosis consistently followed by aneurysm involution during several months. Two of 3 patients who had CN III palsy recovered rapidly, with the third patient (patient 5) with a giant 44-mm aneurysm displacing CN III and the adjacent brain stem. Patient 3 , who also presented with long-standing brain stem dysfunction related to a particularly large aneurysm, showed stabilization, but not complete resolution of index symptoms as well. Based on our results we advocate that clinical recovery parallels to a large degree the degree and duration of neural compression, and the specific sensitivity of the involved neural structures to compression. In conclusion, therapeutic parent artery sacrifice may allow more immediate mass effect reduction, but the benefit of more rapid decompression remains, to our knowledge, unproven. Also, deconstructive methods fundamentally rely on the competence of the collateral arterial supply, ${ }^{11}$ and ischemic complications following therapeutic parent vessel sacrifice include hemianopia or thalamic perforator stroke and certainly do occur as illustrated in On-line Table $1^{1,5,10-15,24}$ Based on our experience, we hence propose that endoluminal reconstruction with the PED likely falls within the range of deconstructive methods in terms of relieving mass effect but offers the benefits of preserved anterograde flow in the parent PCA.

Nonsaccular proximal PCA aneurysms are formidable lesions that remain challenging to treat with any existing method. Despite the encouraging results reported in the present series, there remain limits with the currently available generation of PED. The potentially fatal incident that occurred in patient 3 illustrates how PED deployment becomes progressively more challenging in the more tortuous distal territories, in part due to the relative stiffness of the currently available PED delivery platform. This point is underscored by the extremely complex vascular geometry, the large size of the aneurysm $(31 \mathrm{~mm})$, and the fact that a single 35-mm PED was used in patient 3. Although in our case, full deployment of the PED covered the site of arterial injury and hence was an immediate bailout, future device development will need to address the limitations of the current generation of PED. Depending on the local vascular geometry, we also advocate, in selected cases, overlapping 2 shorter, hence more flex- 
ible devices, which are less prone to torsion during deployment devices, in a manner maintaining single coverage of the normal arterial segments proximal and distal to the aneurysm while maximizing selective coverage across the aneurysm neck (eg, illustrative case, patient 4).

\section{CONCLUSIONS}

PED embolization of nonsaccular aneurysms arising from the proximal PCA compares favorably with historical treatment options in terms of occlusion rate, margin of safety, and neurologic outcome. The present series lends support to the use of the PED, under appropriate clinical circumstances, as a first-line strategy in carefully chosen lesions.

Disclosures: Daniel W. Zumofen—UNRELATED: Employment: New York University School of Medicine, Department of Radiology, Comments: fellow salary for my fellow/clinical instructor position in the Department of Radiology, New York University School of Medicine, New York, New York; Grants/Grants Pending: personal scholarship from the Fund Helmut Hartweg and the Swiss Academy of Medical Science, Comments: 1-year personal scholarship from the Fund Helmut Hartweg and the Swiss Academy of Medical Science to cofinance my fellowship at the New York University School of Medicine. Maksim Shapiro-UNRELATED: Consultancy: Covidien, Comments: I am a Pipeline device proctor and consultant with Covidien; Payment for Development of Educational Presentations: Covidien, Comments: I am a Pipeline device proctor and consultant with Covidien. Tibor Becske-UNRELATED: Consultancy: Covidien/ev3 (consultant); Payment for Lectures (including service on Speakers Bureaus): Covidien/ev3, Comments: I have given lectures for honoraria in the past; Payment for Development of Educational Presentations: Covidien/ev3, Comments: I participated in developing training programs for US physicians in the use of the Pipeline device. Peter K. Nelson—RELATED: Consulting Fee or Honorarium: Covidien; UNRELATED: Consultancy: Covidien.

\section{REFERENCES}

1. Drake CG, Peerless SJ. Giant fusiform intracranial aneurysms: review of 120 patients treated surgically from 1965 to 1992. J Neurosurg 1997;87:141-62

2. Mizutani T, Miki Y, Kojima H, et al. Proposed classification of nonatherosclerotic cerebral fusiform and dissecting aneurysms. Neurosurgery 1999;45:253-59; discussion 259-60

3. de Sousa AA, Dantas FL, Neto AP, et al. Giant posterior cerebral artery aneurysm in a 4-year-old child: case report. Surg Neurol 1996;45:31-35

4. Zeal AA, Rhoton AL Jr. Microsurgical anatomy of the posterior cerebral artery. J Neurosurg 1978;48:534-59

5. Ciceri EF, Klucznik RP, Grossman RG, et al. Aneurysms of the posterior cerebral artery: classification and endovascular treatment. AJNR Am J Neuroradiol 2001;22:27-34

6. Pia HW, Fontana H. Aneurysms of the posterior cerebral artery. Locations and clinical pictures. Acta Neurochir (Wien) 1977;38: 13-35

7. Fischer S, Perez MA, Kurre W, et al. Pipeline embolization device for the treatment of intra- and extracranial fusiform and dissecting aneurysms: initial experience and long-term follow-up. Neurosurgery 2014;75:364-74; discussion 374

8. Pistocchi S, Blanc R, Bartolini B, et al. Flow diverters at and beyond the level of the circle of Willis for the treatment of intracranial aneurysms. Stroke 2012;43:1032-38

9. Phillips TJ, Wenderoth JD, Phatouros CC, et al. Safety of the Pipeline embolization device in treatment of posterior circulation aneurysms. AJNR Am J Neuroradiol 2012;33:1225-31

10. Hallacq P, Piotin M, Moret J. Endovascular occlusion of the posterior cerebral artery for the treatment of P2 segment aneurysms: retrospective review of a 10-year series. AJNR Am J Neuroradiol 2002;23:1128-36
11. Chao KH, Riina HA, Heier L, et al. Endovascular management of dolichoectasia of the posterior cerebral artery report. AJNR Am J Neuroradiol 2004;25:1790-91

12. van Rooij WJ, Sluzewski M, Beute GN. Endovascular treatment of posterior cerebral artery aneurysms. AJNR Am J Neuroradiol 2006;27:300-05

13. Cotroneo E, Gigli R, Guglielmi G. Endovascular occlusion of the posterior cerebral artery in the treatment of P2 ruptured aneurysms. Interv Neuroradiol 2007;13:127-32

14. Roh HG, Kim SS, Han H, et al. Endovascular treatment of posterior cerebral artery aneurysms using detachable coils. Neuroradiology 2008;50:237-42

15. Kocaeli H, Chaalala C, Abruzzo TA, et al. Results of surgical management for posterior cerebral artery aneurysms: 7-year experience in the endovascular era. Acta Neurochir (Wien) 2009;151:1583-91

16. Shapiro M, Raz E, Becske T, et al. Variable porosity of the Pipeline embolization device in straight and curved vessels: a guide for optimal deployment strategy. AJNR Am J Neuroradiol 2014;35:727-33

17. Nelson PK, Lylyk P, Szikora I, et al. The Pipeline embolization device for the intracranial treatment of aneurysms trial. AJNR Am J Neuroradiol 2011;32:34-40

18. Becske T, Kallmes DF, Saatci I, et al. Pipeline for uncoilable or failed aneurysms: results from a multicenter clinical trial. Radiology 2013;267:858-68

19. Siddiqui AH, Abla AA, Kan P, et al. Panacea or problem: flow diverters in the treatment of symptomatic large or giant fusiform vertebrobasilar aneurysms. J Neurosurg 2012;116:1258-66

20. Shapiro M, Raz E, Becske T, et al. Building multidevice Pipeline constructs of favorable metal coverage: a practical guide. AJNR AmJ Neuroradiol 2014;35:1556-61

21. Klisch J, Turk A, Turner R, et al. Very late thrombosis of flow-diverting constructs after the treatment of large fusiform posterior circulation aneurysms. AJNR Am J Neuroradiol 2011;32:627-32

22. Szikora I, Marosfoi M, Salomvary B, et al. Resolution of mass effect and compression symptoms following endoluminal flow diversion for the treatment of intracranial aneurysms. AJNR Am J Neuroradiol 2013;34:935-39

23. Hammoud D, Gailloud P, Olivi A, et al. Acute vasogenic edema induced by thrombosis of a giant intracranial aneurysm: a cause of pseudostroke after therapeutic occlusion of the parent vessel. AJNR Am J Neuroradiol 2003;24:1237-39

24. Liu L, He H, Jiang C, et al. Deliberate parent artery occlusion for non-saccular posterior cerebral artery aneurysms. Interv Neuroradiol 2011;17:159-68

25. Yonekawa Y, Roth P, Fandino J, et al. Aneurysms of the posterior cerebral artery and approach selection in their microsurgical treatment: emphasis on the approaches-SAHEA and SCTTA. Acta Neurochir Suppl 2011;112:85-92

26. Lee JY, Kwon BJ, Kang HS, et al. Subarachnoid hemorrhage from a dissecting aneurysm of the posterior cerebral artery in a child: rebleeding after stent-assisted coiling followed by stent-within-stent technique. J Korean Neurosurg Soc 2011;49:134-38

27. Wang H, Du R, Stary J, et al. Dissecting aneurysms of the posterior cerebral artery: current endovascular/surgical evaluation and treatment strategies. Neurosurgery 2012;70:1581-88; discussion 1588

28. Briganti F, Napoli M, Tortora F, et al. Italian multicenter experience with flow-diverter devices for intracranial unruptured aneurysm treatment with periprocedural complications-a retrospective data analysis. Neuroradiology 2013;54:1145-52

29. Jennett B, Bond M. Assessment of outcome after severe brain damage. Lancet 1975;1:480-84

30. Farrell B, Godwin J, Richards S, et al. The United Kingdom transient ischaemic attack (UK-TIA) aspirin trial: final results. J Neurol Neurosurg Psychiatry 1991;54:1044-54 